SUPPLEMENTARY DATA

\title{
Structure and activity of the N-terminal region of the eukaryotic cytolysin equinatoxin II
}

\author{
Alison DRECHSLER ${ }^{* \dagger}$, Cristina POTRICH ${ }^{\ddagger}$, Jennifer K. SABO*, Mattia FRISANCO ${ }^{\S}$, \\ Graziano GUELLA ${ }^{\S}$, Mauro DALLA SERRA ${ }^{\ddagger}$, Gregor ANDERLUH", Frances \\ SEPAROVIC ${ }^{\dagger}$ and Raymond S. NORTON $* 1$ \\ * The Walter \& Eliza Hall Institute of Medical Research, 1G Royal Parade, Parkville, VIC \\ 3050, Australia, ${ }^{\dagger}$ School of Chemistry, University of Melbourne, VIC 3010, Australia, ${ }^{\ddagger}$ ITC- \\ CNR, Institute of Biophysics, Via Sommarive 18, 38050 Povo (Trento) Italy, ${ }^{\S}$ Laboratory of \\ Bioorganic Chemistry, Department of Physics, University of Trento, Via Sommarive 14, \\ 38050 Povo (Trento), Italy, "Department of Biology, Biotechnical Faculty, University of \\ Ljubljana, Vecna pot 111, 1000 Ljubljana, Slovenia \\ ${ }^{1}$ To whom correspondence should be addressed (Phone: +6139345 2306. Fax: +6139345 \\ 2686. E-mail: Ray.Norton@wehi.edu.au)
}


Table S1. ${ }^{1} \mathrm{H}$ and ${ }^{13} \mathrm{C}$ chemical shifts (ppm) of EqTII $11-32$ in DMSO- ${ }^{2} \mathrm{H}_{6}$ at $30{ }^{\circ} \mathrm{C}$

\begin{tabular}{|c|c|c|c|c|c|c|}
\hline Residue & NH & $\mathrm{H} \alpha\left({ }^{13} \mathrm{C} \alpha\right)$ & $\mathbf{H} \beta$ & $\mathbf{H} \gamma$ & Others & $\begin{array}{c}\Delta \delta / \Delta \mathrm{T} \\
\left(\mathrm{ppb} /{ }^{\circ} \mathrm{C}\right)\end{array}$ \\
\hline 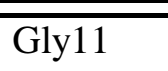 & 8.12 & 3.56 & 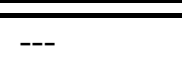 & 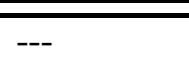 & 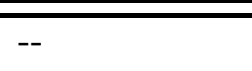 & \\
\hline Ala12 & 8.57 & $4.48(47.7)$ & 1.23 & --- & --- & -6.1 \\
\hline Ser13 & 8.18 & $4.31(54.6)$ & 3.57 & --- & $5.01 \mathrm{OH}$ & -9.5 \\
\hline Leu14 & 8.07 & $4.11(51.4)$ & 1.49 & 1.60 & $0.83,0.87 *$ & \\
\hline Ser 15 & 7.98 & $4.24(54.7)$ & 3.53 & -- & $5.01 \mathrm{OH}$ & -8.0 \\
\hline Phe16 & 7.95 & $4.49(53.5)$ & $3.05 / 2.80$ & -- & $7.15-7.25$ & \\
\hline Asp17 & 8.35 & $4.59(49.2)$ & $2.68 / 2.50$ & -- & & -8.0 \\
\hline Ile18 & 7.76 & $4.12(57.6)$ & 1.72 & $1.42,1.10$ & $0.80,0.79$ & \\
\hline Leu19 & 7.91 & $4.33(50.7)$ & 1.49 & 1.60 & $0.83,0.87^{*}$ & \\
\hline Lys20 & 8.02 & $4.20(52.1)$ & $1.75 / 1.61$ & 1.36 & $1.56,2.75^{\dagger}$ & \\
\hline Thr21 & 7.68 & $4.23(58.0)$ & 3.97 & 1.03 & $4.93 \mathrm{OH}$ & \\
\hline Val22 & 7.70 & $4.12(58.8)$ & 2.01 & $0.83,0.84$ & & \\
\hline Leu23 & 8.00 & $4.25(50.7)$ & 1.49 & 1.60 & $0.83,0.87$ & \\
\hline Glu24 & 7.89 & $4.20(51.6)$ & $1.83 / 1.74$ & 2.23 & & -7.5 \\
\hline Ala25 & 7.97 & $4.25(48.0)$ & 1.21 & -- & & \\
\hline Leu26 & 7.92 & $4.25(50.9)$ & 1.49 & 1.60 & $0.83,0.87 *$ & \\
\hline Gly27 & 8.07 & $3.74 / 3.67(41.6)$ & -- & & & \\
\hline Asn 28 & 8.09 & $4.62(49.2)$ & $2.58 / 2.44$ & -- & $7.44,6.91$ & $\begin{array}{l}-5.9,-5.0 \\
\left(\mathrm{CONH}_{2}\right)\end{array}$ \\
\hline Val 29 & 7.70 & $4.12(57.6)$ & 2.01 & $0.83,0.84$ & & \\
\hline Lys30 & 7.71 & $4.21(52.1)$ & 1.70 & 1.34 & $1.56,2.76 \dagger$ & \\
\hline $\operatorname{Arg} 31$ & 7.84 & $4.25(52.2)$ & 1.70 & 1.50 & $3.08,7.56 \mathrm{NH}$ & $\begin{array}{ll}-6.5 & (-2.9 \\
\left.\delta_{\mathrm{NH}}\right) & \end{array}$ \\
\hline Lys32 & 7.70 & $4.11(51.4)$ & 1.70 & 1.34 & $1.56,2.76 \dagger$ & \\
\hline
\end{tabular}

The peak assignments of Leu14,19 and $26\left(^{*}\right)$ and Lys20, 30 and $32\left(^{\dagger}\right)$ are ambiguous because of spectral overlap. 
Table S2. ${ }^{1} \mathrm{H}$ chemical shifts and ${ }^{3} J_{\mathrm{HNH} \alpha}$ of $\mathrm{EqTII}_{1-32}$ in $125 \mathrm{mM} \mathrm{DPC}-{ }^{2} \mathrm{H}_{38}$ in $95 \% \mathrm{H}_{2} \mathrm{O} / 5 \%$ ${ }^{2} \mathrm{H}_{2} \mathrm{O}$ at $\mathrm{pH} 5.1$ and $45{ }^{\circ} \mathrm{C}$.

\begin{tabular}{|c|c|c|c|c|c|c|}
\hline Residue & $\mathrm{C}^{\alpha}$ & $\mathrm{C}^{\beta}$ & $\mathrm{NH}$ & $\mathrm{C}^{\alpha} \mathrm{H}$ & $\mathrm{C}^{\beta} \mathrm{H}$ & Other $\mathrm{H}$ \\
\hline$\overline{\text { Ser1 }}$ & 57.3 & 63.4 & & 4.17 & $4.00^{*}$ & \\
\hline Ala2 & 52.8 & & & 4.42 & $1.42^{\dagger}$ & \\
\hline Asp3 & & & 8.31 & 4.65 & $2.63,2.74$ & \\
\hline Val4 & & & 8.08 & 4.04 & 2.13 & $\mathrm{C}^{\gamma} \mathrm{H}_{3} 0.95^{\ddagger}$ \\
\hline Ala5 & 55.0 & & 8.33 & 4.22 & $1.42^{\dagger}$ & \\
\hline Gly6 & & & 8.21 & $3.90,3.90$ & & \\
\hline Ala7 & 53.5 & & 7.95 & 4.35 & $1.43^{\dagger}$ & \\
\hline Val8 & 62.3 & 39.2 & 8.03 & 4.04 & 2.13 & $\mathrm{C}^{\gamma} \mathrm{H}_{3} 0.95^{\ddagger}$ \\
\hline Ile9 & & & 8.00 & 4.13 & 1.90 & $\begin{array}{l}\mathrm{C}^{\gamma} \mathrm{H}_{2} 1.19,1.54, \mathrm{C}^{\gamma} \mathrm{H}_{3} 0.92^{\S}, \\
\mathrm{C}^{\delta} \mathrm{H}_{3} 0.84\end{array}$ \\
\hline Asp10 & & & 8.26 & 4.60 & $2.73^{*}$ & \\
\hline Gly11 & & & 8.41 & $3.88,3.88$ & & \\
\hline Ala12 & & & 8.14 & 4.31 & $1.45^{\dagger}$ & \\
\hline Ser13 & & 63.9 & 8.09 & 4.43 & $3.93^{*}$ & \\
\hline Leu14 & 56.4 & 41.7 & 8.06 & 4.35 & $2.00^{*}$ & $\mathrm{C}^{\gamma} \mathrm{H} 1.56, \mathrm{C}^{\delta} \mathrm{H}_{3} 0.94^{\mathbb{q}}$ \\
\hline Ser15 & & 64.5 & 7.72 & 4.48 & $3.84^{*}$ & \\
\hline Phe16 & 59.7 & 39.3 & 8.65 & 4.38 & 3.22 & $\left(\mathrm{C}^{\varepsilon} \mathrm{H}_{2}, \mathrm{C}^{\delta} \mathrm{H}_{2}, \mathrm{C}^{\zeta} \mathrm{H}\right) 7.32^{* *}$ \\
\hline Asp17 & 54.6 & & 8.36 & 4.43 & $2.74^{*}$ & \\
\hline Ile18 & & 38.3 & 8.04 & 3.91 & 2.01 & $\begin{array}{l}\mathrm{C}^{\gamma} \mathrm{H}_{2} 1.28,1.71, \mathrm{C}^{\gamma} \mathrm{H}_{3} 0.95^{\S}, \\
\mathrm{C}^{\delta} \mathrm{H}_{3} 0.78\end{array}$ \\
\hline Leu19 & & 42.0 & 7.81 & 3.95 & $1.75^{*}$ & $\mathrm{C}^{\gamma} \mathrm{H} 1.75, \mathrm{C}^{\delta} \mathrm{H}_{3} 0.91,0.94^{\|}$ \\
\hline Lys 20 & 60.6 & & 8.14 & 3.85 & $1.84,1.88$ & $\begin{array}{l}\mathrm{C}^{\gamma} \mathrm{H}_{2} 1.38,1.47, \\
\mathrm{C}^{\delta} \mathrm{H}_{2} 1.72, \mathrm{C}^{\varepsilon} \mathrm{H}_{2} 2.74^{\dagger \dagger} \mathrm{N}^{\zeta} \mathrm{H}_{3}-{ }_{-} \S\end{array}$ \\
\hline Thr21 & & 68.9 & 7.73 & 3.99 & 4.36 & $\mathrm{C}^{\gamma} \mathrm{H}_{3} 1.28 \|$ \\
\hline Val22 & 67.2 & 31.9 & 8.07 & 3.62 & 2.28 & $\mathrm{C}^{\gamma} \mathrm{H}_{3} 0.93,1.07^{\S}$ \\
\hline Leu23 & 58.6 & & 8.28 & 4.01 & $1.88^{*}$ & $\mathrm{C}^{\gamma} \mathrm{H} 1.58, \mathrm{C}^{\delta} \mathrm{H}_{3} 0.90^{\mathbb{I}}$ \\
\hline Glu24 & 59.0 & & 8.18 & 4.02 & $2.10,2.20$ & $\mathrm{C}^{\gamma} \mathrm{H}_{2} 2.29,2.51$ \\
\hline Ala25 & 55.0 & 18.8 & 7.86 & 4.24 & $1.56^{\dagger}$ & \\
\hline Leu26 & & & 8.08 & 4.15 & $1.88,1.94$ & $\mathrm{C}^{\gamma} \mathrm{H} 1.60, \mathrm{C}^{\delta} \mathrm{H}_{3} 0.89,1.05^{\|}$ \\
\hline Gly27 & & & 7.96 & $3.89,3.95$ & & \\
\hline Asn 28 & 54.1 & & 7.80 & 4.77 & $2.81,2.93$ & $\mathrm{~N}^{\gamma} \mathrm{H}_{2} 6.91,7.59$ \\
\hline Val 29 & 32.5 & & 7.66 & 4.04 & 2.21 & $\mathrm{C}^{\gamma} \mathrm{H}_{3} 0.98,1.06^{\S}$ \\
\hline Lys30 & 57.2 & 33.3 & 8.04 & 4.38 & $1.78,1.88$ & 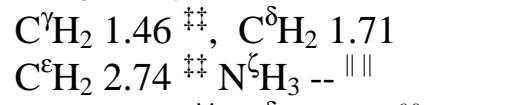 \\
\hline Arg31 & 56.5 & 31.3 & 8.19 & 4.34 & $1,80,1.90$ & 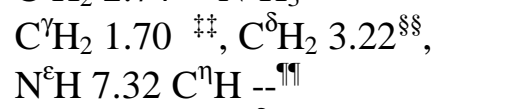 \\
\hline Lys 32 & 57.7 & 34.0 & 7.81 & 4.15 & $1.75,1.82$ & $\begin{array}{l}\mathrm{C}^{\gamma} \mathrm{H}_{2} 1.45^{*+}, \mathrm{C}^{\delta} \mathrm{H}_{2} 1.70^{\S \S}, \\
\mathrm{C}^{\varepsilon} \mathrm{H}_{2--}{ }^{* * *}, \mathrm{~N}^{\zeta} \mathrm{H}_{3}--\|\|\end{array}$ \\
\hline
\end{tabular}

${ }^{1} \mathrm{H}$ chemical shifts were degenerate for ${ }^{*} \mathrm{H} \beta 2$ and $\mathrm{H} \beta 3$ of Ser1, 13 and 15, Asp10 and 17, and Leu 14, 19 and 23, ${ }^{\dagger}$ methyl $\mathrm{H} \beta$ of Ala2, 5, 7, 12 and 25, ${ }^{\ddagger}$ methyl $\mathrm{H} \gamma$ of Val4 and 8, ${ }^{\S}$ methyl H $\gamma$ of Val 22 and 29 and Ile9 and 18, "methyl H $\delta$ of Ile 9 and 18, Leu19 and 26, and The 21, II methyl $\mathrm{H} \delta$ of Leu14 and 23, ${ }^{* *}$ aromatic protons of Phe16, ${ }^{\dagger \dagger} \mathrm{H} \delta 2$ and $\mathrm{H} \delta 3$ and $\mathrm{H} \varepsilon 2$ and 
Hع3 peaks of Lys20, and 30, ${ }^{*}$ the $\mathrm{H} \gamma 2$ and $\mathrm{H} \gamma 3$ peaks of Lys30 and 32, ${ }^{\S} \mathrm{H} \delta 2$ and $\mathrm{H} \delta 3$ of Arg31 and Lys32

Resonances were not observed for ${ }^{\|\|} \mathrm{N}^{\zeta} \mathrm{H}_{3}$ of Lys20, 30 and 32 and, " ${ }^{\text {qII }} 4 \mathrm{H \eta}$ of Arg 31 and *** $\mathrm{H} \varepsilon$ of Lys 32 
Table S3. Coupling constants, backbone amide temperature coefficients, amide exchange data, H-bonds evident in structure, and residue solvent accessibility of EqTII $1-32$.

\begin{tabular}{|c|c|c|c|c|c|c|c|}
\hline Residue $^{*}$ & ${ }^{3} \mathbf{J}_{\text {HNHA }}$ & $\begin{array}{c}\Delta \delta / \Delta \mathbf{T} \\
\left(\mathbf{p p b} /{ }^{\circ} \mathbf{C}\right)^{\dagger}\end{array}$ & Exchange & $\begin{array}{l}\text { H-bond } \\
\text { acceptor }\end{array}$ & $\begin{array}{c}\text { \% Solvent } \\
\text { accessibility }\end{array}$ & $\phi$ angle ${ }^{\mathbb{T}}$ & $\psi$ angle ${ }^{\pi}$ \\
\hline Ser 1 & & - & & & $73 \pm 11$ & & \\
\hline Ala 2 & & - & & & $48 \pm 10$ & $-82 \pm 14$ & \\
\hline Asp 3 & & -6.5 & & & $48 \pm 12$ & $\begin{array}{c}-109 \pm \\
38\end{array}$ & \\
\hline Val 4 & & -7.5 & $S$ & & $47 \pm 9$ & $-98 \pm 22$ & \\
\hline Ala 5 & $<6 \mathrm{~Hz}$ & -8.5 & & & $34 \pm 12$ & & \\
\hline Gly 6 & & -5.0 & & & $24 \pm 14$ & $-69 \pm 18$ & \\
\hline Ala 7 & & -4.5 & & & $29 \pm 10$ & $-85 \pm 16$ & \\
\hline Val 8 & & -7.0 & & & $46 \pm 7$ & $-79 \pm 23$ & \\
\hline Ile 9 & & -8.5 & $S$ & & $47 \pm 8$ & $-83 \pm 12$ & $-15 \pm 20$ \\
\hline Asp 10 & & -8.5 & & & $39 \pm 13$ & $-63 \pm 9$ & $-37 \pm 17$ \\
\hline Gly 11 & & -6.0 & & & $20 \pm 9$ & & \\
\hline Ala 12 & $<6 \mathrm{~Hz}$ & -4.5 & & & $30 \pm 11$ & & \\
\hline Ser 13 & & -5.5 & & & $32 \pm 8$ & & \\
\hline Leu 14 & & - & & & $43 \pm 12$ & $-66 \pm 10$ & \\
\hline Ser 15 & & -2.5 & & 11 & $31 \pm 5$ & $-80 \pm 18$ & $-25 \pm 21$ \\
\hline Phe 16 & & -5.0 & & & $48 \pm 8$ & $-64 \pm 9$ & \\
\hline Asp 17 & & -4.0 & & 13 & $33 \pm 7$ & $-71 \pm 12$ & \\
\hline Ile 18 & & -5.0 & & & $37 \pm 10$ & & \\
\hline Leu 19 & & -5.0 & & & $42 \pm 6$ & $-63 \pm 4$ & \\
\hline Lys 20 & & -5.5 & & & $38 \pm 11$ & $-63 \pm 4$ & \\
\hline Thr 21 & & -3.5 & & 17 & $33 \pm 6$ & $-65 \pm 5$ & \\
\hline Val 22 & & -6.0 & $S$ & 18 & $33 \pm 7$ & & \\
\hline Leu 23 & & -6.5 & $S$ & 19 & $43 \pm 6$ & & \\
\hline Glu 24 & & -4.0 & & & $41 \pm 8$ & $-66 \pm 5$ & \\
\hline Ala 25 & & -1.5 & & 21 & $24 \pm 7$ & & \\
\hline Leu 26 & & -5.0 & $\mathrm{~S}$ & & $39 \pm 7$ & $-77 \pm 14$ & \\
\hline Gly 27 & & -3.5 & & 23 & $24 \pm 8$ & & \\
\hline Asn 28 & & -1.0 & & 24 & $41 \pm 13$ & & \\
\hline Val 29 & & -1.5 & $\mathrm{~S}$ & 25 & $44 \pm 6$ & $-82 \pm 20$ & \\
\hline Lys 30 & & -8.5 & & & $53 \pm 6$ & $-75 \pm 29$ & \\
\hline Arg 31 & & -7.5 & $\mathrm{~S}$ & & $58 \pm 9$ & $-70 \pm 11$ & \\
\hline Lys 32 & & -7.0 & $\mathrm{~s}$ & & $75 \pm 1$ & & \\
\hline
\end{tabular}

${ }^{*}$ Residue numbering of EqTII ${ }_{1-32}$ sequence as shown in the sequence in Fig 1. ${ }^{\dagger}$ Measured over the temperature range 25 to $45^{\circ} \mathrm{C}$ at $\mathrm{pH} 5.1{ }^{*} \mathrm{~S}$ denotes $\mathrm{NH}$ observed in $1 \mathrm{D}{ }^{1} \mathrm{H}$ spectra after $2 \mathrm{~h}$ in ${ }^{2} \mathrm{H}_{2} \mathrm{O}$ at $\mathrm{pH} 4.8$ and $35^{\circ} \mathrm{C}$. ${ }^{\S}$ Potential $\mathrm{H}$-bond acceptors based on the final family 
of structures. " Solvent accessibility measured for each residue in MOLMOL (1) across the family of 20 structures and ${ }^{\text {II }} \phi$ and $\psi$ angles were calculated using the TALOS program.

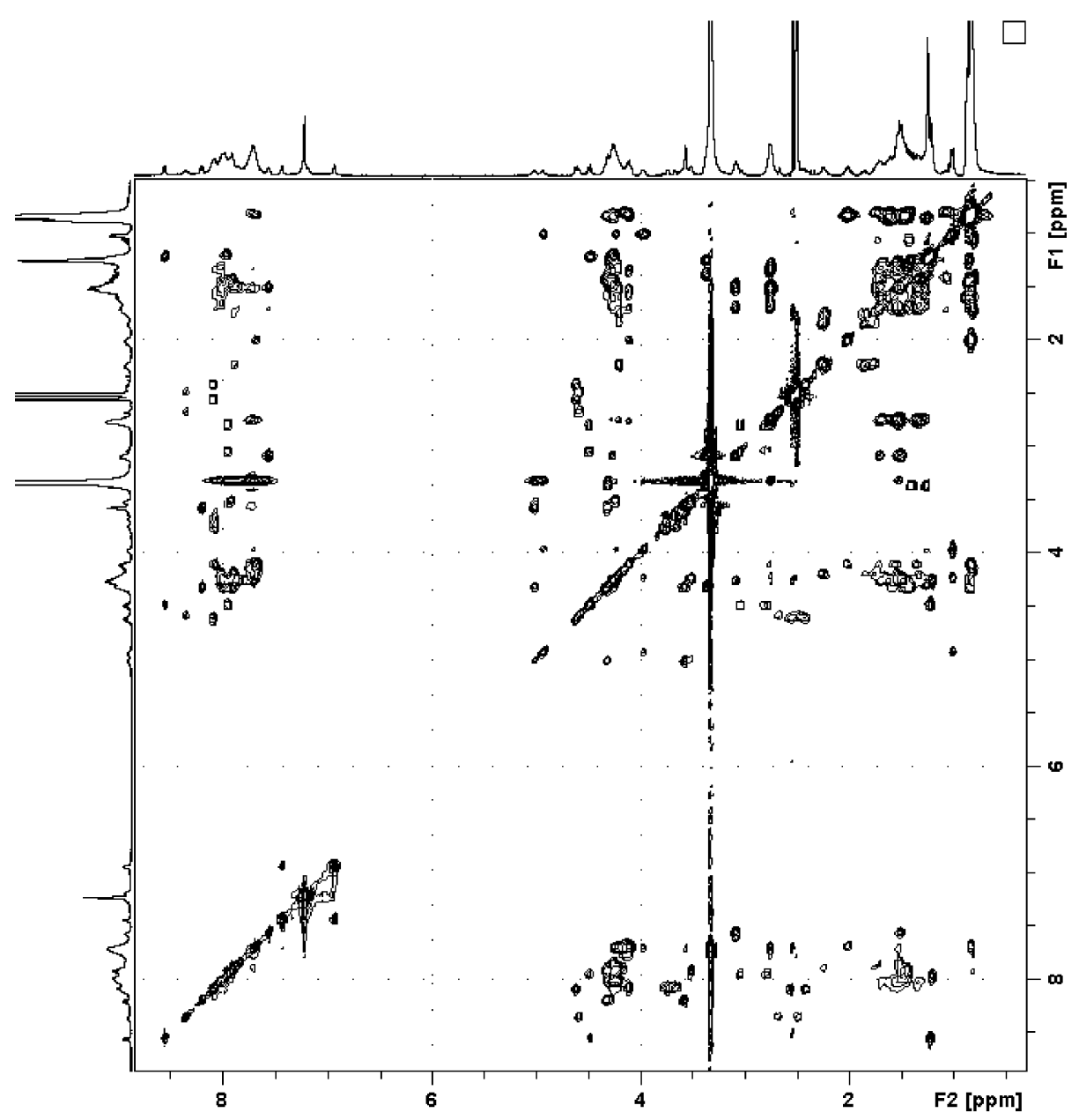

Figure S1. TOCSY spectrum of EqTII $11-32$ in DMSO- ${ }^{2} \mathrm{H}_{6}$ at $30{ }^{\circ} \mathrm{C}$ 


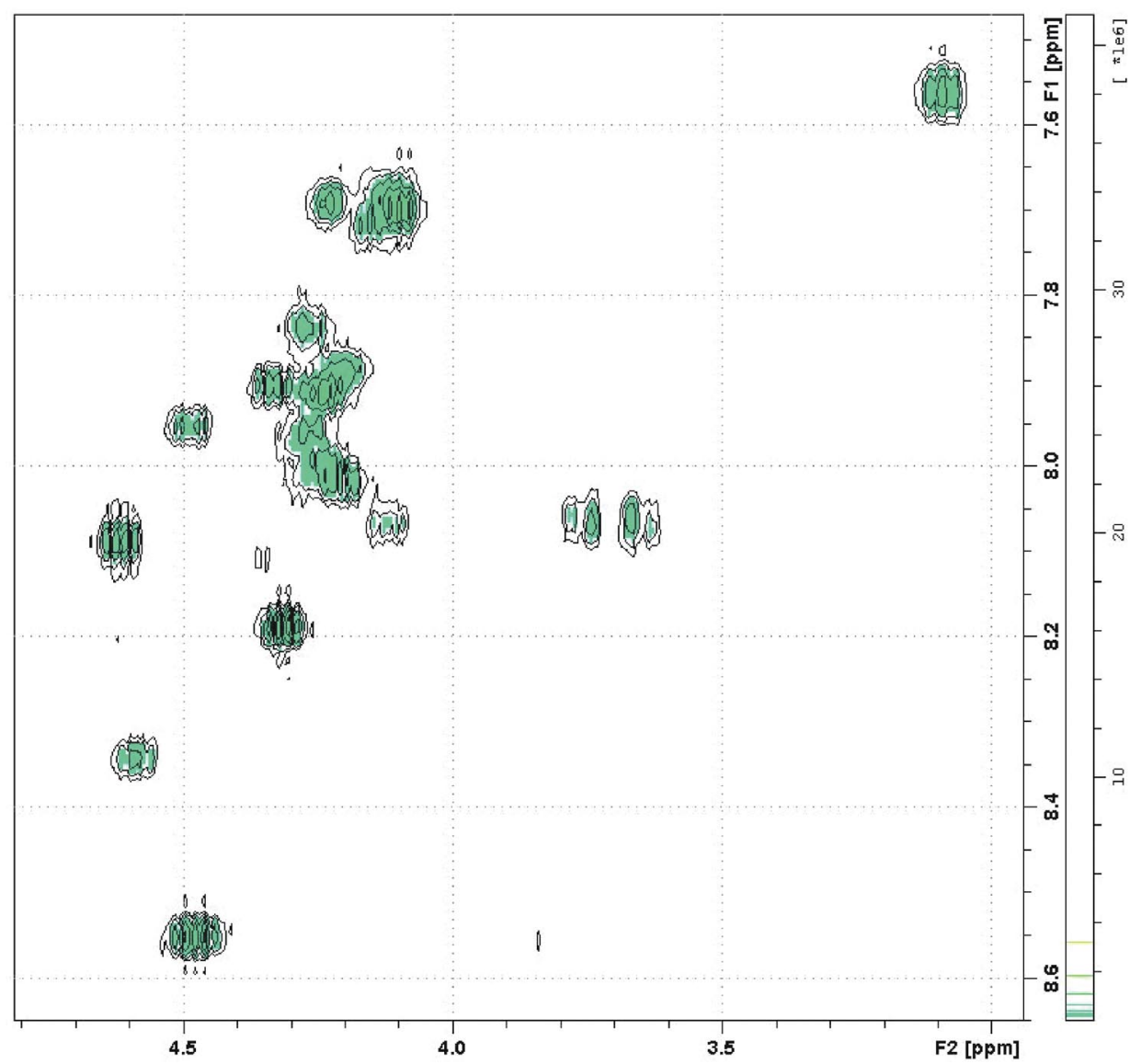

Figure S2. NH-H ${ }^{\alpha}$ cross-peaks in the DQF-COSY spectrum of EqTII ${ }_{11-32}$ in DMSO- ${ }^{2} \mathrm{H}_{6}$ at $30^{\circ} \mathrm{C}$ 


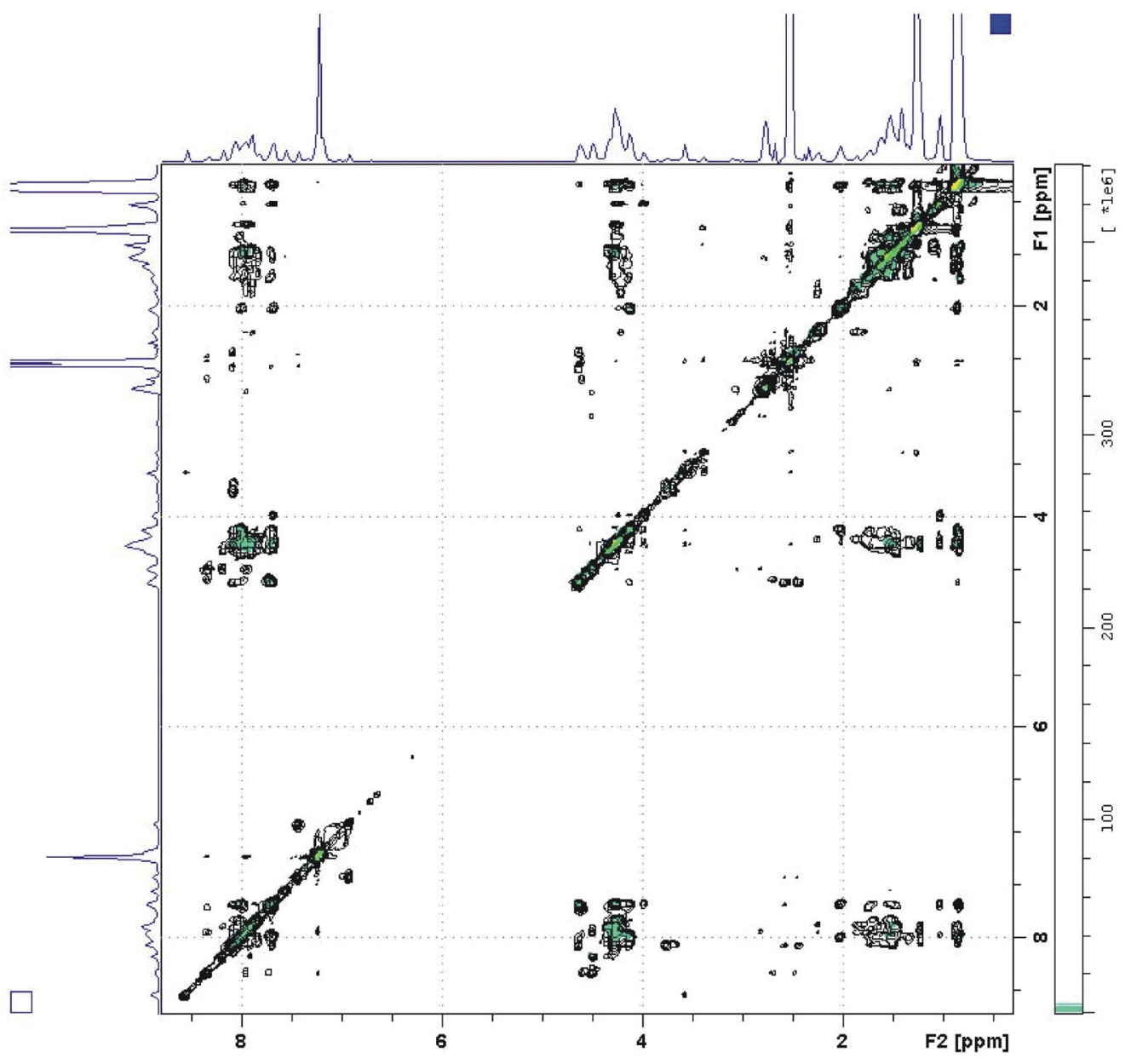

Figure S3. NOESY spectrum (mixing time $600 \mathrm{~ms}$ ) of EqTII ${ }_{11-32}$ in DMSO- ${ }^{2} \mathrm{H}_{6}$ at $30{ }^{\circ} \mathrm{C}$. 
A)

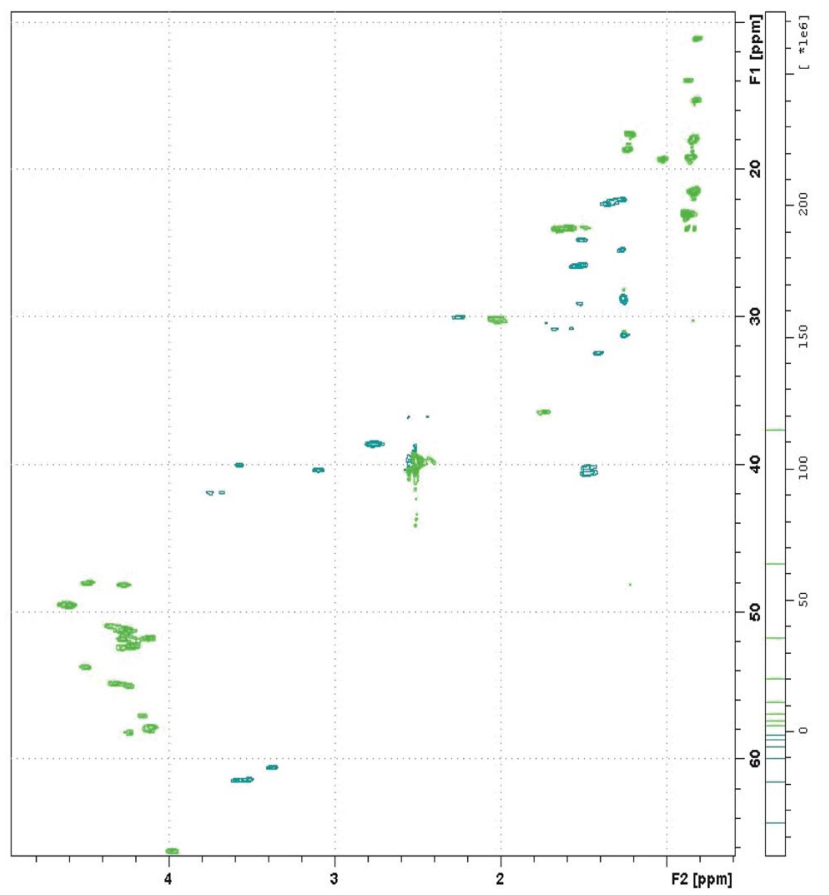

B)

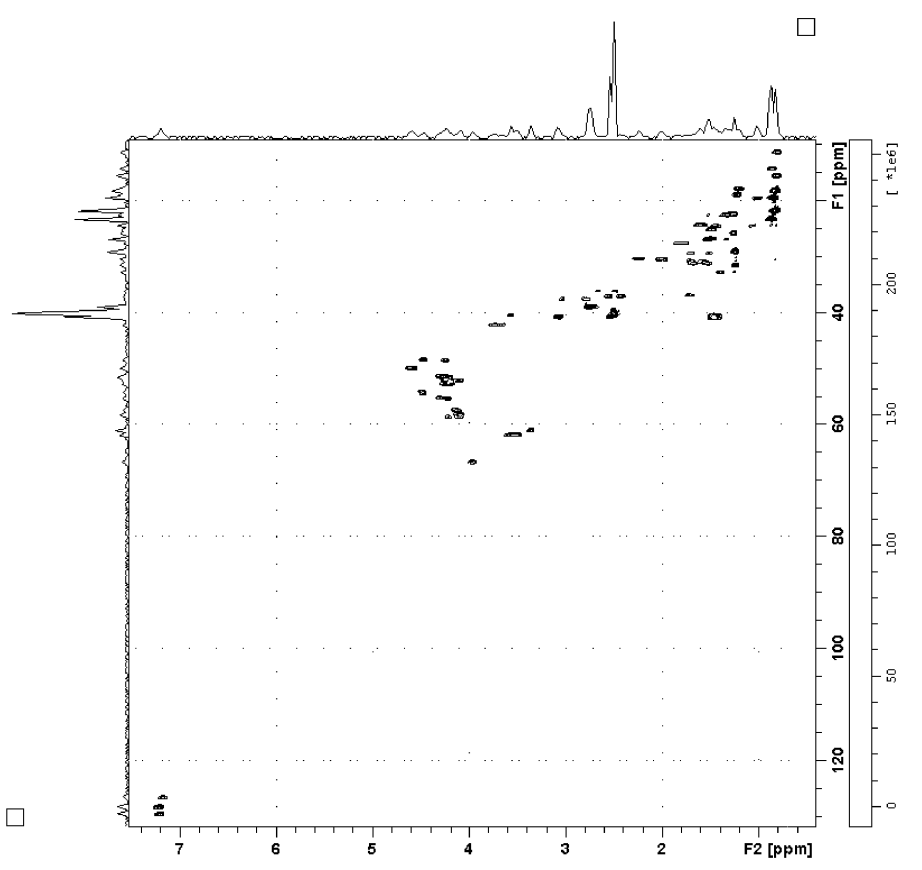

Figure S4. EqTII ${ }_{11-32}$ in DMSO- ${ }^{2} \mathrm{H}_{6}$ at $30{ }^{\circ} \mathrm{C}$. (A) Upfield region of ${ }^{13} \mathrm{C}$ HSQC spectrum (B) Entire ${ }^{13} \mathrm{C}$ HSQC spectrum 

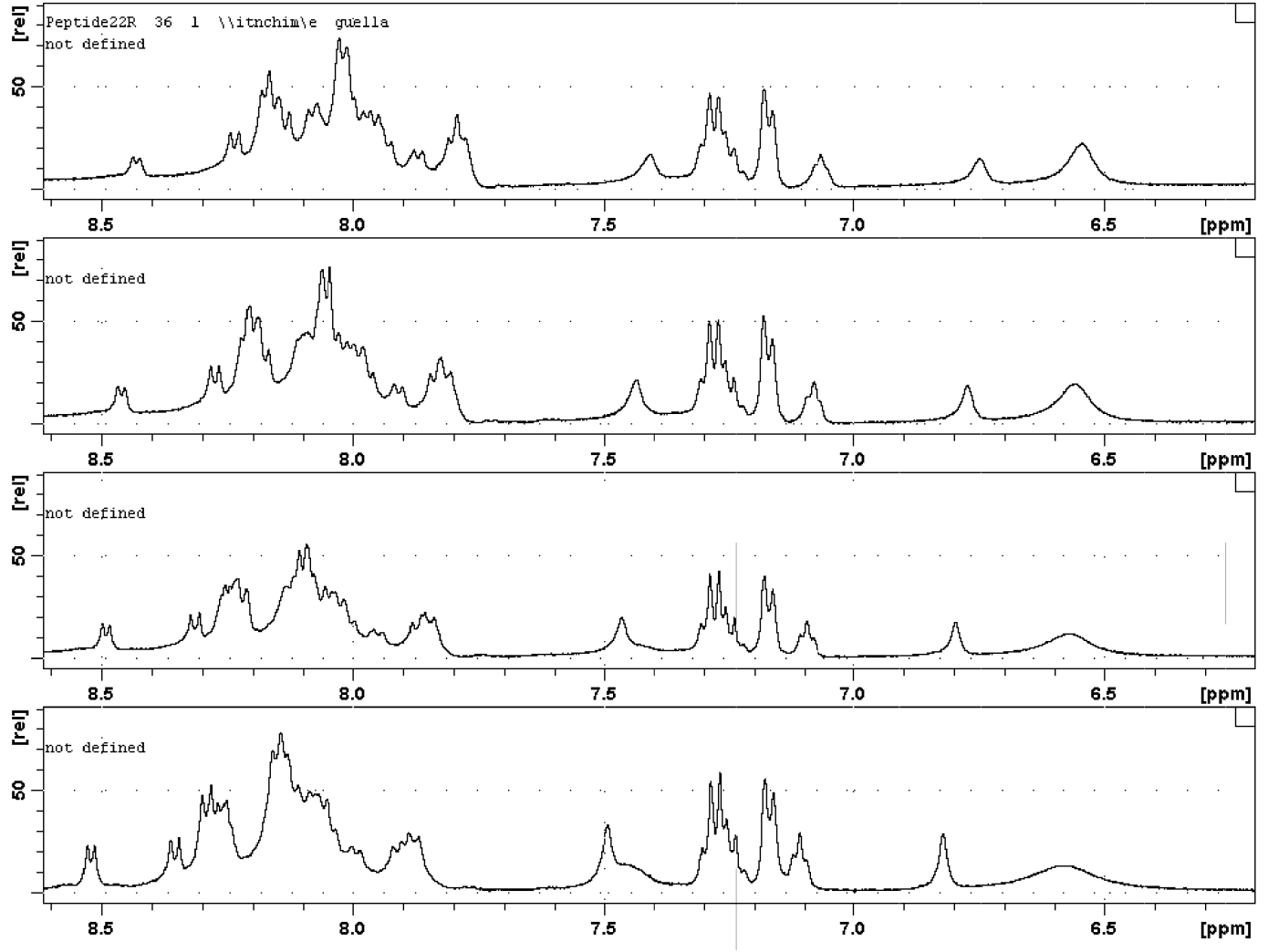

Figure S5. Temperature effect on ${ }^{1} \mathrm{H}$ spectra of EqTII ${ }_{11-32}$ in DMSO- ${ }^{2} \mathrm{H}_{6}$ over the range $25-40{ }^{\circ} \mathrm{C}$ 


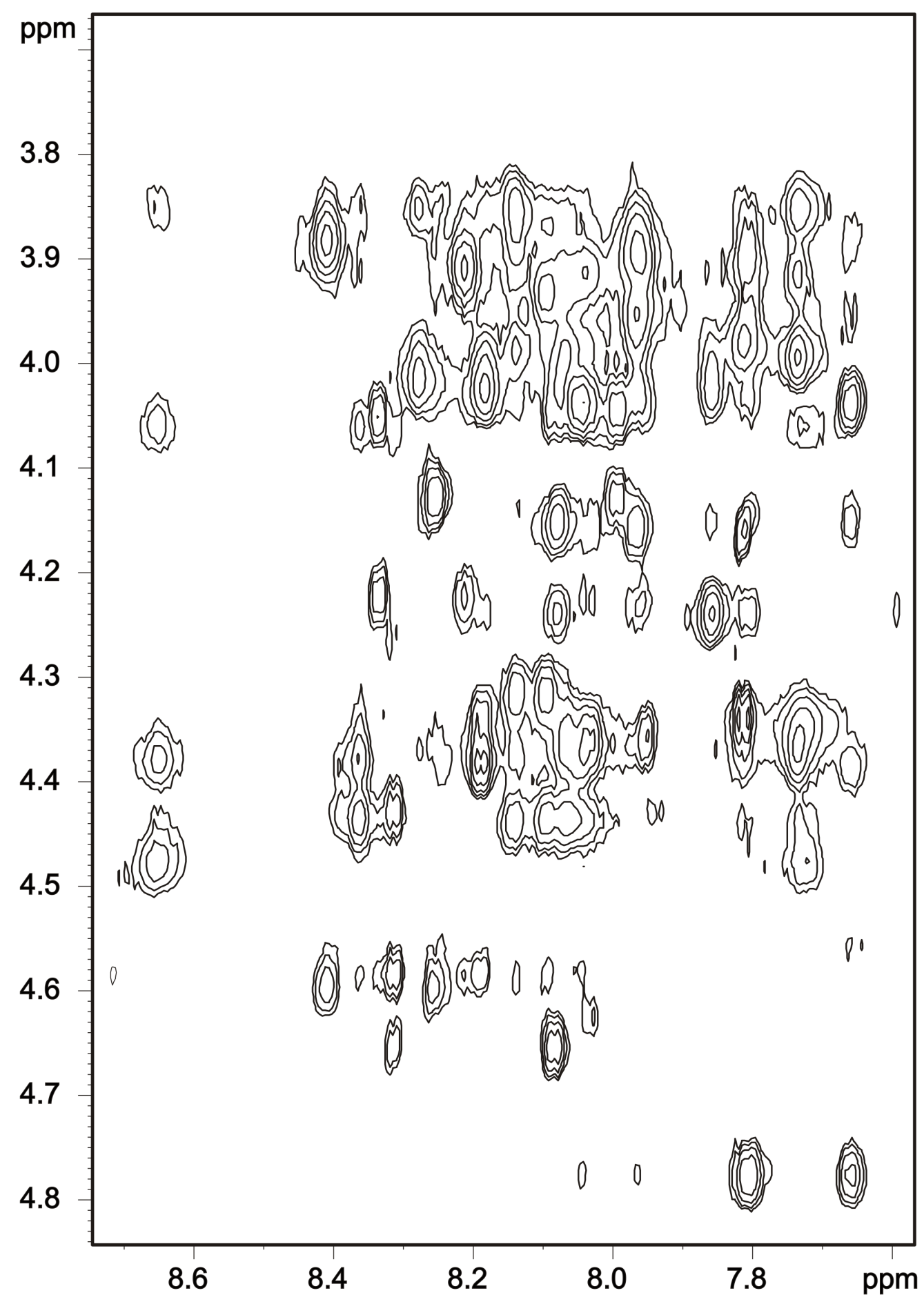

Figure S6. NH-CH region of $600 \mathrm{MHz}$ NOESY spectrum of EqTII ${ }_{1-32}$ in DPC micelles at pH 5.1 and $45^{\circ} \mathrm{C}$. 

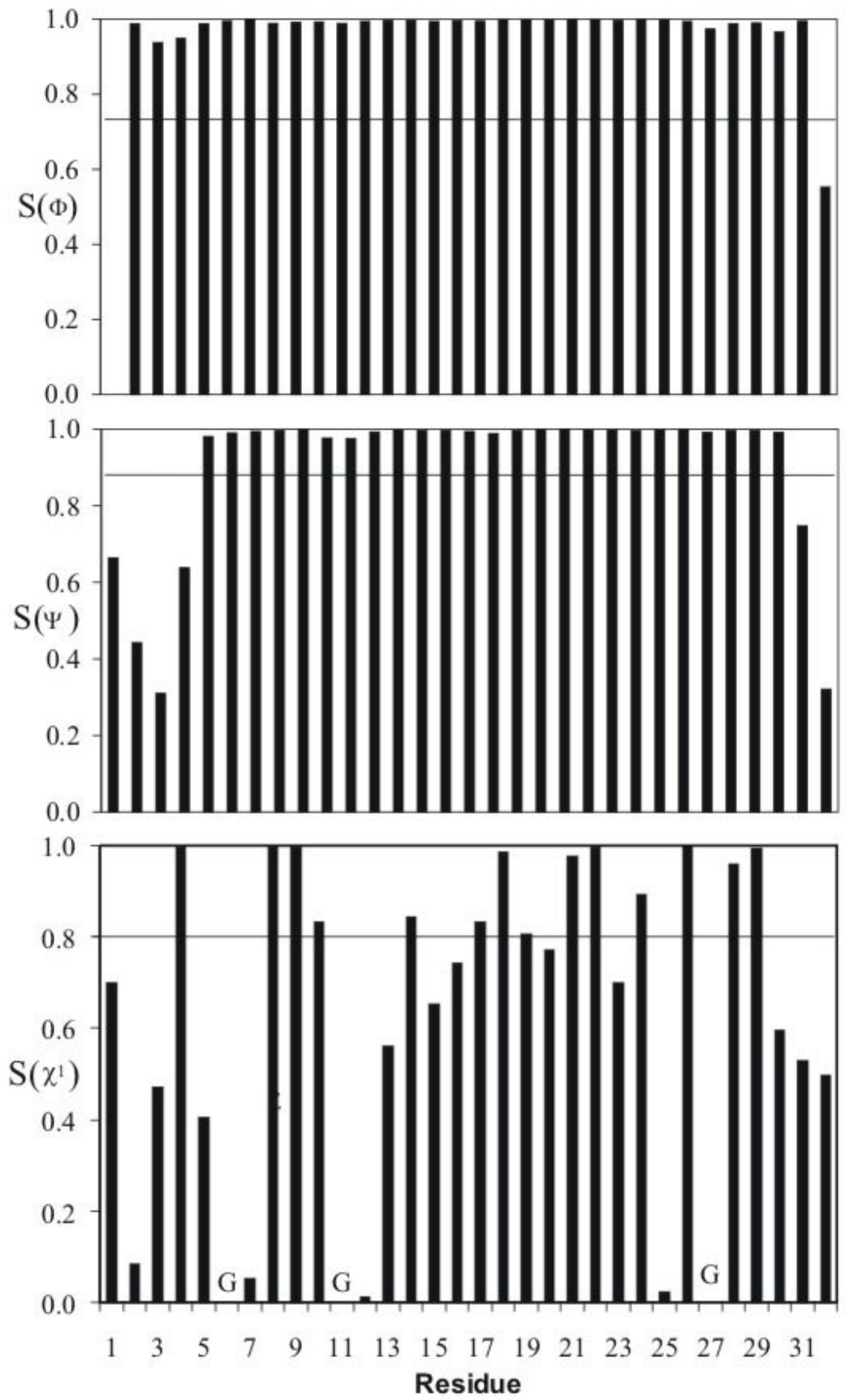

Figure S7. Angular order parameters, $S(\phi), S(\psi)$ and $S\left(\chi_{1}\right)$, as a function of residue number for $\mathrm{EqTII}_{1-32}$. Angular order parameters were calculated using the program MOLMOL (1). 

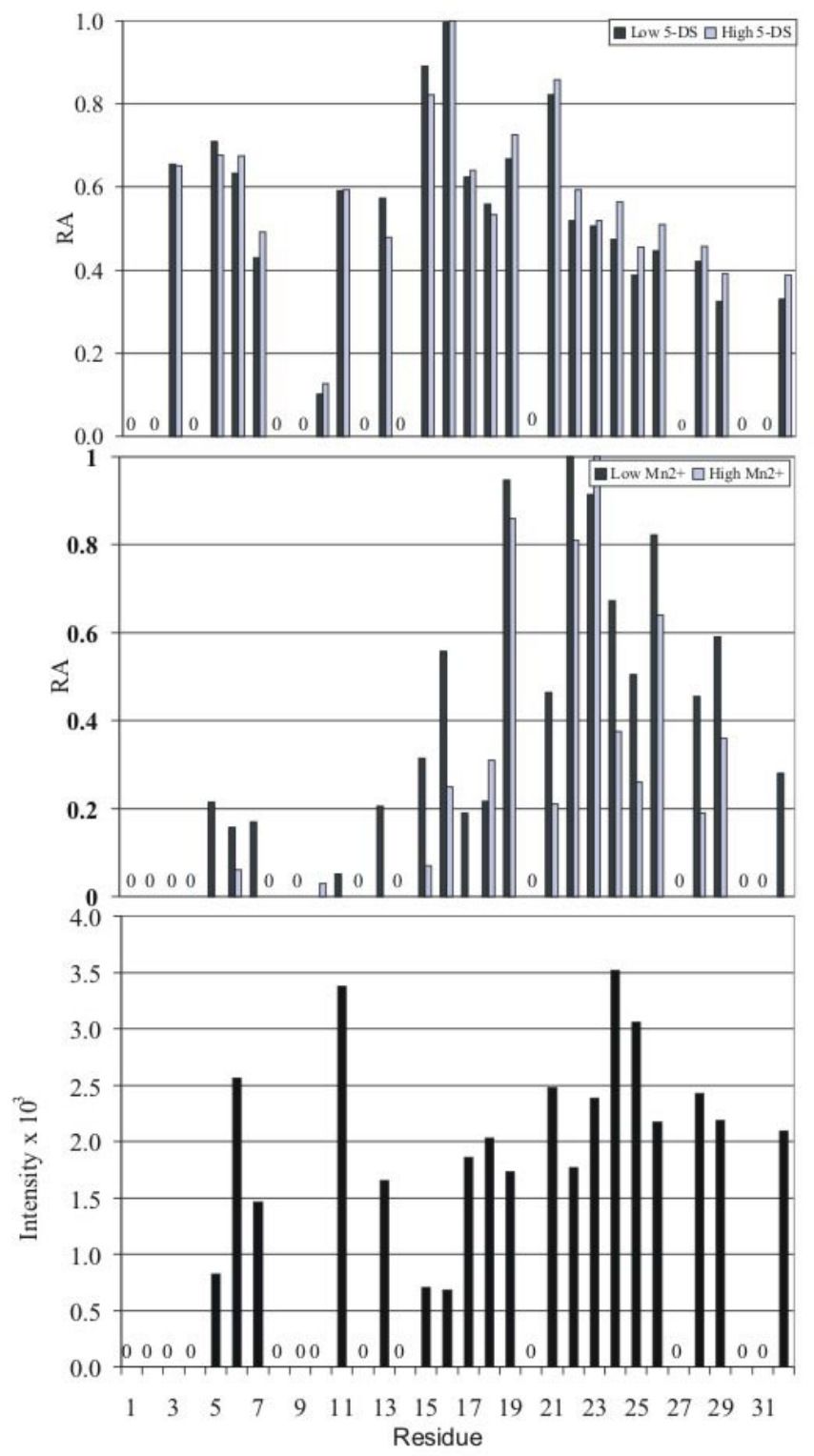

Figure S8. Change in cross-peak intensity for EqTII ${ }_{1-32}$ upon addition of A) the micelleinserted probe 5-d-SPPC, and B) the solvent-exposed probe $\mathrm{Mn}^{2+}$. C) Relative intensity of each peak in the absence of either probe. Changes in intensity could not be determined because of peak overlap or absence of NOE for residues marked with 0 .

\section{REFERENCE}

1. Koradi, R., Billeter, M. and Wüthrich, K. (1996) MOLMOL: a program for display and analysis of macromolecular structures. Journal of Molecular Graphics. 14, 51-55 